Meta

Journal des tradlucteurs

Translators' Journal

\title{
Besoins et ressources en terminologie dans cinq domaines
}

\section{Ad Hermans}

Volume 37, numéro 2, juin 1992

URI : https://id.erudit.org/iderudit/003063ar

DOI : https://doi.org/10.7202/003063ar

Aller au sommaire du numéro

Éditeur(s)

Les Presses de l'Université de Montréal

\section{ISSN}

0026-0452 (imprimé)

1492-1421 (numérique)

Découvrir la revue

Citer cet article

Hermans, A. (1992). Besoins et ressources en terminologie dans cinq domaines. Meta, 37(2), 316-331. https://doi.org/10.7202/003063ar d'utilisation que vous pouvez consulter en ligne.

https://apropos.erudit.org/fr/usagers/politique-dutilisation/ 


\section{ÉTUDES TERMINOLOGIQUES ET LINGUISTIQUES}

\section{BESOINS ET RESSOURCES EN TERMINOLOGIE DANS CINQ DOMAINES}

Le Centre de terminologie de Bruxelles (Institut libre Marie-Haps) a été chargé par le Service de la langue française de l'exécutif de la Communauté française de Belgique d'une enquête complémentaire à celle qui avait été effectuée par la Section arts et sciences de la communication de l'Université de Liège. Il s'agissait de dresser un inventaire précis des ressources et des besoins en terminologie et en néologie dans cinq domaines sélectionnés.

Une préenquête demandée par le Service de la langue française avait permis au centre de dresser une liste de domaines prioritaires en Belgique pour l'activité terminologique.

Sur la base de ces données, il a été convenu avec le Service de la langue française que la recherche se concentrerait sur cinq domaines constituant un volet important et des langues de spécialités, et de la vie économique belge :

- la bureautique : source importante de textes édités et traduits; cette sous-langue technique est très utilisée parce que c'est par elle que se vulgarise l'informatique;

- la robotique: une des technologies nouvelles les plus dynamiques. Les robots posent des problèmes liés à l'interdisciplinarité en même temps qu'à la nouveauté;

- la chimie: un des créneaux belges et un domaine omniprésent dans tous les autres secteurs économiques. Les apports français y sont importants;

- la pharmacologie : un autre créneau belge, également source de publications multiples et pour lequel des investissements sont faits dans la communication;

- l'architecture et la construction : ce domaine d'activités est économiquement porteur et le savoir-faire y est ancien.

Dans un premier temps, une exploration des cinq domaines et de leur langue a été effectuée sur la base de lectures et d'entretiens avec des spécialistes.

Ensuite, la terminologie «blanche» et «grise» y a été recherchée, et ce sur la base de sept sources: traducteurs indépendants, bureaux et services de traduction, centres de recherche, centres de documentation et courtiers en informations documentaires, rédaction des revues scientifiques et techniques éditées en Belgique, bibliographies, entreprises, organisations professionnelles, ministères et organismes d'État.

Une étude documentaire et linguistique des cinq domaines a alors été menée en collaboration avec des experts. Pour cette étude des besoins et des ressources autres que les vocabulaires (publiés ou non), les catégories d'interlocuteurs suivantes ont été selectionnées:

- auteurs et/ou rédacteurs d'un grand nombre de documents susceptibles de guider l'usage; auteurs de rapports de recherche et d'ouvrages théoriques;

- traducteurs de documents susceptibles d'influencer l'usage ou représentant un segment important du marché de la traduction ;

- diffuseurs des termes de la spécialité: éditeurs de revues; journalistes de revues techniques, rédacteurs d'agences de presse spécialisées, professeurs d'université ;

- observateurs possédant une connaissance globale du domaine et de sa langue : organismes ministériels de promotion de l'industrie du domaine, associations professionnelles, centres de recherche, grands «consommateurs»;

- organismes détenteurs de corpora ou d'informations à propos des catégories précédentes : centres de documentation, librairies, bibliothèques. 
Sur la base des renseignements obtenus au cours d'entretiens préliminaires, nous avons décidé de rédiger un questionnaire complémentaire à celui de la recherche de l'Université de Liège sur les besoins et les ressources en terminologie et de l'envoyer à l'ensemble des traducteurs belges.

Ce questionnaire visait surtout l'obtention de renseignements à propos des facteurs suivants, lesquels exercent une influence sur la qualité des glossaires éventuellement faits par les traducteurs et donc sur leur utilité pour d'autres :

- la documentation technique (dictionnaires, notamment les «bibles» du domaine, revues et monographies spécialisées, documents rédigés en parallèle dans plusieurs langues) utilisée par ces traducteurs et leur appréciation de cette documentation ;

- les méthodes de dépouillement de ces documents ;

- les autres sources de néologie;

- l'évolution du vocabulaire observée par le traducteur dans ses différents domaines de spécialité, notamment le rythme d'apparition des néologismes. Bien que les cinq domaines aient été mentionnés explicitement dans le questionnaire, nous avons inclus également dans ce dernier tous les autres domaines scientifiques et techniques, afin de ne perdre aucune information utile.

Le questionnaire a été envoyé entre le 10 et le 15 mai à 739 traducteurs de Bruxelles et de Wallonie. Nous n'avons reçu qu'une trentaine de réponses, le questionnaire ayant croisé un très gros questionnaire de l'Université de Mons fait sous les auspices de la Chambre des traducteurs, interprètes et philologues ainsi qu'un questionnaire sur les spécialités des traducteurs et sur leur expérience professionnelle élaboré par un bureau de traduction.

Cent quinze traducteurs et une cinquantaine de bureaux de traduction ont alors été recontactés par téléphone et interrogés plus brièvement à l'aide d'un guide d'interview. Thème de l'interview : les pratiques du traducteur en matière de recherche documentaire et de consignation de termes. Si le traducteur faisait des fiches de terminologie, nous lui demandions leur objet: compléter les dictionnaires spécialisés existants, couvrir exhaustivement un domaine ou consigner simplement les termes inconnus de leur auteur (que ces termes soient ou non dans les dictionnaires).

Au cours d'entretiens approfondis avec des experts des différents domaines, nous avons posé des questions sur la vie du vocabulaire de leur spécialité : documents-clés dans les différents types de communication pratiqués par la discipline, notamment les textes importants pour l'entrée des néologismes; rythme d'apparition des nouveaux termes (et de disparition de vocables) ; sous-domaines dans lesquels ces néologismes sont les plus fréquents ; évolution de la discipline et de son vocabulaire.

Un guide d'interview similaire à celui qui avait été utilisé pour les centres de recherche a été élaboré pour les entreprises. Lorsqu'il n'y avait pas de traducteur et que les traductions étaient faites dans la firme, nous demandions également qui se chargeait de les faire.

Aux professeurs des établissements d'enseignement, nous avons demandé la langue dans laquelle sont présentées les notions techniques aux étudiants et celle dans laquelle les étudiants effectuent leurs lectures. Nous leurs avons en outre posé des questions sur les cours d'anglais dispensés aux étudiants.

\section{LA TERMINOLOGIE DE LARCHITECTURE ET DE LA CONSTRUCTION}

Le domaine du bâtiment est généralement abordé à trois niveaux différents : l'industrie du bâtiment (l'exécution); la construction (l'étude du détail) et l'architecture (la conception). L'architecture, qui se situe le plus en amont, intègre la construction mais 
également d'autres éléments comme la fonction et la forme. Le vocabulaire de l'architecture assimile donc le vocabulaire de la construction, mais il bénéficie également d'autres apports et les intègre tous en fonction de ses critères propres.

L'activité constructive utilise quant à elle un grand nombre de techniques, aux origines très diversifiées, qui se doivent en outre de s'adapter aux conditions très particulières du travail en chantier. Cette adaptation entraîne des modifications de ces techniques qui peuvent être reconnues comme des techniques à part entière.

Les spécialistes ne distinguent pas seulement le vocabulaire de l'architecture de celui de la construction; ils séparent la terminologie des techniques convoquées de celle des chantiers. Dans ces deux secteurs, les mêmes procédés et matériaux peuvent avoir des dénominations différentes, ce qui prête parfois à confusion. Le vocabulaire technique peut être normalisé ou non, mais il prend généralement place dans le système constructif conventionnel ou prescriptif.

Le vocabulaire constructif est donc interdisciplinaire (produits chimiques - en nombre croissant -, produits métallurgiques, vocabulaire scientifique de la physique thermique et acoustique, etc.), mais il est spécifique.

L'évolution récente du vocabulaire de la construction épouse évidemment l'évolution technique, qui fait largement appel à d'autres domaines. Compte tenu de la variété et de la complexité croissante de ces domaines, l'assimilation des nouveaux termes ne se réalise malheureusement plus aussi facilement que par le passé. C'est notamment le cas des nouveaux matériaux de synthèse (avec leurs abréviations), qui ont tendance à être confondus. On devrait par exemple dénoncer l'usage, en voie de généralisation, de la dénomination Roofing pour désigner des membranes qui ne répondent pas du tout à la définition normalisée de ce terme.

Par ailleurs, la part croissante prise par les composants fabriqués en usine entraîne le recours de plus en plus fréquent aux éponymes pour désigner les produits. Ainsi vélux pour fenêtre rampante, lexan pour polycarbonate, plexiglas pour poly-méthylméthaérylate, shunt pour conduit collectif à branchements unitaires (alors que la firme Shunt, qui a créé ce type de conduits, utilisé même à l'étranger, a disparu depuis de nombreuses années !), derbigum pour désigner en vrac toutes les nouvelles membranes de bitum-polymère, qu'elles soient à base d'élastomères ou de plastomères. Il est à remarquer que le recours aux éponymes est rigoureusement prohibé dans les marchés de l'État mais qu'il est par contre fréquent dans les documents prescriptifs des marchés privés.

D'autre part, la multiplication des intervenants a diversifié les points de vue et les dénominations d'une notion : un même élément peut être baptisé différemment selon le point de vue envisagé. Ainsi, le contreplaqué du concepteur désigne le multiplex de l'exécutant.

Enfin, le recours naturel et fréquent, en Belgique, à l'importante littérature technique française (notamment aux normes, beaucoup plus nombreuses que les nôtres) est lui aussi source de confusions. C'est ainsi que, dans le domaine de la sécurité, le degré de résistance au feu (RF), défini dans les normes belges, devient très souvent le degré coupe-feu (CF) français, qui n'a pas la même signification. On trouve également souvent le degré de réaction au feu français (M4 par exemple) au lieu du degré belge (A4), qui s'appuie à la fois sur la classifícation française et la classification anglaise. On trouve aussi la mention du degré pare-flammes, alors que cette notion, reconnue en France et dans d'autres pays, n'est pas admise en Belgique.

En architecture et en construction, les ressources terminologiques sont abondantes.

Les documents comme les normes, les Cahiers des charges-types du ministère des Travaux publics, les Notes d'information technique du CSTC, les Spécifications 
techniques de l'Institut national du logement, les Agréments techniques de l'UBATC, les Tables $B B / S f B$ et les Cahiers de FAB/CSTC proposent un vocabulaire précis et normalisé en français et en néerlandais. Pour les rédactions et traductions dans ces domaines, on se réfêre à ces documents, dont le vocabulaire et les informations techniques sont complets. Comme ils sont bilingues, ils sont utilisés comme ressources terminologiques lorsqu'ils comportent des listes de termes.

Les Spécifications techniques unifiées sont de vrais documents terminologiques qui offrent des définitions précises des termes. Selon les experts consultés, ces définitions sont complètes et exactes, mais elles reflètent une terminologie de conception et non de mise en cuvre (on y trouve par exemple les subdivisions du réseau d'égout mais pas les éléments constructifs nécessaires à sa réalisation).

Il existe également une dizaine de dictionnaires spécialisés (qui sont mentionnés dans l'inventaire) et un grand nombre de documents utilisables et utilisés comme dictionnaires ou glossaires, tels que des thésaurus et les agendas du bâtiment. Nous avons aussi relevé un certain nombre de lexiques à usage privé.

Contrairement à ce qui se passe pour d'autres domaines étudiés, nous n'avons pas trouvé de bureaux de traduction spécialisés en architecture et construction. Les textes d'architecture (articles de revues ou ouvrages) sont souvent traduits par des architectes. On traduit relativement peu en architecture (les architectes aiment les plans et les photos, à partir desquels ils rendent leurs impressions personnelles). En outre, la spécificité de l'architecture dans les différents pays limite la circulation d'une série de textes à l'échelon international. Les textes techniques sont rédigés et traduits par des bureaux de conception et d'études qui disposent, grâce aux documents de référence (Cahier des charges-type, normes, etc.), de la terminologie nécessaire.

En construction, les traductions des revues techniques sont souvent faites par des traducteurs employés depuis longtemps par des centres de recherche (et qui disposent donc d'«informants» sur place). Les traductions sont faites par les bureaux d'études et, pour les devis, par les soumissionnaires (ingénieurs dans les grandes firmes, techniciens dans les PME) de la langue en question. Dans certains cas (notamment lorsque le temps manque), certains bureaux font appel aux services de traducteurs et des firmes ou revues font traduire ponctuellement quelques articles. Les traductions sont donc souvent faites par des professionnels du bâtiment ou des bureaux d'études.

Les nombreuses publications techniques en deux langues portant sur l'architecture et la construction constituent des sources terminologiques intéressantes si leur auteur les a rédigées dans sa langue maternelle.

$\mathrm{Si}$, en construction, le bilinguisme des locuteurs leur fait choisir des termes anglais en lieu et place des termes français existants (sprinkler pour asperseur), il ne semble pas y avoir de problème majeur d'emprunts à l'anglais dans l'art de bâtir.

D'une façon générale, la terminologie de la construction est assez bien régulée grâce aux travaux et documents d'organismes reconnus comme le CSTC.

En architecture, on assiste à la disparition d'une partie du vocabulaire ancien (suite aux simplifications du modernisme, notamment), alors que ces vocables restent pertinents et redeviennent même indispensables, avec le retour d'une plus grande variété du «langage» architectural (pour les façades, par exemple). Un dictionnaire thématique visuel rendrait d'appréciables services, notamment en expertise.

La préoccupation principale des rédacteurs en architecture n'est pas de pouvoir disposer de glossaires (surtout pas du type terme à terme), mais de gérer d'une manière efficace la masse des documents en circulation. D'où le nombre élevé de thésaurus et d'organismes d'information et de documentation. 
La terminologie en architecture est bien d'expression française et belge. La Belgique fait par exemple partie des «pays de la brique» (à la différence de la France, qui découvre actuellement le parement en briques), mais elle est, avec les États-Unis, le seul pays où l'on construit les murs extérieurs depuis l'intérieur du bâtiment. Cette particularité est à l'origine de toute une terminologie technique spécifique différente de celle qui est utilisée en France. Il apparaît néanmoins que des glossaires de termes en français et en néerlandais comportant des définitions techniques et juridiques, comme ceux que l'on trouve dans les STS, répondent à un besoin des architectes et des entrepreneurs.

L'évolution de la terminologie est plus rapide pour les matériaux de construction et les noms de produits, mais, en ce domaine, la réglementation (notamment par le ministère des Travaux publics), les exigences de précision de la terminologie des contrats et le fonctionnement des centres de recherche para-étatiques garantissent une terminologie précise, en français comme en néerlandais.

Les entreprises de construction qui travaillent souvent à l'étranger constituent un cas à part. Elles ont besoin d'une terminologie multilingue, souvent terme à terme (arabeanglais, notamment) pour les contacts de tous les jours. Le dictionnaire de Benefalux offre une terminologie de ce type; il comporte des termes techniques, surtout de chantier. Ces termes ne sont cependant pas très spécialisés.

\section{LA TERMINOLOGIE DE L'INFORMATIQUE}

L'informatique est un domaine immense et qui s'accroît sans cesse, imposant non seulement ses machines mais aussi ses méthodes ${ }^{1}$, et donc peut-être une partie de son vocabulaire, aux disciplines qu'elle touche.

En informatique, la Belgique vend plus de produits qu'elle ne fait de recherches. À part ceux des universités, il n'existe apparemment pas de centres de recherche paraétatiques analogues à ceux qu'on trouve pour la construction, par exemple.

Les publications scientifiques ne sont pas nombreuses : il n'existe pas de collection «Informatique» chez les éditeurs scientifiques belges ni de grande revue scientifique belge d'informatique (analogue à la revue française Rairo de l'AFCET). Malgré les accords-cadres négociés avec des maisons d'édition françaises d'informatique (par exemple les Presses universitaires de Bruxelles), les chercheurs en informatique préfèrent, dans la majorité des cas, lire et publier en anglais chez les grands éditeurs internationaux, la rédaction en anglais ne leur posant aucun problème.

En Belgique, les publications scientifiques françaises seraient moins lues que les américaines; une des raisons avancées par certains chercheurs et industriels est que les Français s'aligneraient moins sur les normes internationales pour leur produits (notamment pour les télécommunications), ce qui compliquerait l'exportabilité de ces derniers...

Par ailleurs, la diffusion des résultats des recherches belges auprès de tous les chercheurs qu'ils intéresseraient n'est pas assez large. Aussi certaines recherches sontelles menées en parallèle.

Le critère de la langue n'intervient pas dans le choix des ouvrages spécialisés des bibliothèques des facultés et instituts d'informatique : à partir de la seconde candidature, les étudiants lisent couramment l'anglais de leur spécialité et leur demande linguistique est, à ce stade, celle d'une connaissance active (pouvoir parler d'informatique en anglais). L'enseignement de l'informatique est d'ailleurs un véritable cours d'anglais, d'autant plus intensif et efficace qu'il est indirect (l'attention des étudiants est tournée vers l'assimilation des notions, non des dénominations). Les termes sont présentés en deux langues, oralement et dans les syllabus, mais c'est souvent la dénomination anglaise que les professeurs utilisent devant les étudiants. 
En informatique technique des gros systèmes, les lettres d'annonce des constructeurs constituent la voie de pénétration la plus importante pour les néologismes, tant anglais que français, car elles sont le canal d'information principal des informaticiens. Les grands constructeurs publient également des bulletins pour leurs produits de microinformatique et ils éditent également des bulletins spécialisés ${ }^{2}$. Ces publications belges sont en anglais.

En informatique théorique, les publications les plus importantes pour la diffusion des néologismes anglais sont d'abord les actes des grands colloques, souvent publiés chez North-Holland et Springer, puis les rapports de recherche des universités et des centres. Les néologismes français sont créés par les chercheurs qui reprennent et continuent les recherches entamées aux États-Unis et les «vulgarisent» ainsi dans les rapports de recherche de leurs universités et de leurs centres. Il existe également une école française d'informatique théorique (qui publierait entre autres chez Dunod) dans laquelle des Belges seraient actifs.

Pour l'informatique technique et les applications des gros systèmes, les lettres d'annonce, d'abord, les dossiers de presse, ensuite, puis les manuels d'utilisateurs sont les principaux canaux d'information des professionnels. Les fiches techniques des constructeurs sont trop spécialisées pour avoir un impact important sur le vocabulaire.

En micro-informatique technique et d'application, c'est par les dossiers de presse que s'introduisent sans doute les néologismes; la presse et les manuels d'utilisation les vulgarisent ensuite. Dans ce sous-domaine, le rôle des traducteurs (français et, à un degré bien moindre, belges) n'est pas négligeable: c'est sur leurs versions des manuels d'utilisateurs que sont basés les ouvrages de vulgarisation. Les journalistes interviennent également: en micro-informatique, c'est souvent par la presse (traduite ou non) que les annonces sont connues. Par contre, les traducteurs des manuels techniques des gros systèmes n'ont pas beaucoup d'influence sur la langue : la qualité de ces - rares traductions laissant à désirer, les informaticiens leur préfèrent les originaux.

L'enregistrement de la terminologie maison de leur client dans le dossier d'analyse est, pour les sociétés de conception d'applications et de programmes «clés en main», une étape classique du travail d'analyse. Comme celle des bureaux de traduction, cette terminologie n'est malheureusement pas accessible. Ces sociétés donnent des directives pour l'élaboration de la documentation technique afférente aux programmes qu'elles développent ; cette documentation est en anglais, par souci d'harmonie avec l'ensemble des documents conçus pour les gros systèmes. Leurs manuels d'utilisateurs sont très simples et dans la langue du client (leurs applications sont généralement très conviviales et très personnalisées si elles sont destinées à un personnel non informaticien).

Les documents commerciaux, qui constituent la plus grosse part du marché de la traduction, comportent relativement peu de termes techniques (ils s'adressent aux décideurs et mettent donc plutôt l'accent sur les avantages de la technologie décrite que sur ses caractéristiques). Les contrats, devis, plans d'action, guides commerciaux et catalogues de produits sont d'autres documents dont la traduction par les professionnels est fréquente.

Le marché belge de la traduction est assez important pour l'informatique. La concurrence $\mathrm{y}$ est vive.

La terminologie française de l'informatique belge est plus anglicisée que celle de la France, notamment dans les documents techniques, les textes de vulgarisation technique et l'informatique d'application. En Belgique comme en France, la terminologie des gros systèmes est tout à fait anglaise. La francisation progressive de la micro-informatique d'application remontera-t-elle vers les gros systèmes? De grands constructeurs ont essayé de franciser leur environnement (messages et commandes des systèmes d'exploitation 
notamment), ce qui n'est pas sans provoquer la résistance des informaticiens. Le manque de standardisation des mini-ordinateurs (malgré le succès du système d'exploitation UNIX) pourrait bien conférer aux protocoles de télécommunication le statut de norme de fait; ces protocoles sont en anglais.

Néanmoins, les termes français pénètrent progressivement en Belgique, par osmose. En dix ans, les chercheurs en informatique sont passés d'un «bilinguisme passif» à un «bilinguisme actif»: en 1970, un professeur d'université devait réfléchir pour trouver l'équivalent français du terme anglais véhiculant une notion nouvelle pour ses étudiants; actuellement, ce terme se présente immédiatement à son esprit (il utilise la dénomination anglaise avec ses collègues).

Les anglicismes sont dus à divers facteurs :

- un certain effet de repoussoir exercé par la politique de francisation du gouvernement français ;

- la bonne connaissance de l'anglais qu'ont les techniciens et les scientifiques belges. Dans tous les établissements d'enseignement de l'informatique, le cours d'anglais obéit de plus en plus fréquemment aux méthodes $L_{S P}^{3}$, d'où son efficacité : incapables de lire un journal en anglais, les étudiants apprennent sans douleur à écrire en anglais des livres entiers dans leur spécialité ;

- les interférences avec les langues germaniques voisines (le néerlandais et, pour les publications des sociétés Siemens et Nixdorf, par exemple, l'allemand), dont l'emprunt massif à l'anglais est copié comme modèle de néologie par les francophones ;

- le fait qu'une francisation trop poussée couperait les contacts des informaticiens avec leurs collègues néerlandophones, contacts plus développés qu'avec leurs homologues français ;

- le rôle d'argot, au sens de P. Singy ${ }^{4}$, que joue l'anglais : comme l'argot, le jargon des termes anglais d'informatique a, en sus de ses fonctions de «signum social» (à la fois discriminant et cohésif), d'économie linguistique et d'esthétique (le plaisir de jouer avec les mots), un rôle cryptique lorsqu'il est utilisé par les commerciaux avec leurs clients ;

- le caractère codé du langage informatique, proche de celui des communications aéronautiques (commandes des langages de programmation; utilisation du langage d'intelligence artificielle PROLOG comme outil de logique formelle et de communication des résultats de recherche);

- les mauvaises traductions dont regorge le vocabulaire belge de l'informatique. Dans le traitement de l'information, le principe d'équivalence dynamique énoncé par Nida est régulièrement bafoué ${ }^{5}$ : les termes français ne sont pas imagés car les tropes anglais n'y ont pas été gommés (périphrases, notamment syntagmes descriptifs : protocole d'établissement de liaison traduit piteusement handshaking). À quelques exceptions près (étreinte fatale), ils ne témoignent pas non plus de l'humour (que montre peeckaboo, par exemple) des auteurs de leurs homologues anglo-saxon, ni de leur sens musical (bascule bistable, qui traduit flip-flop, n'a pas son assonance). Les calques syntaxiques sur l'anglais sont fréquents (barres code), notamment les omissions de joncteurs (instruction machine). La cohérence anglaise ne s'y retrouve pas: spooler se traduit par logiciel de distribution de tâches mais spool, par spoule). Les navettes d'emprunts, ou remprunts, de l'anglais au français sont beaucoup plus rares (burotics, maintenance, random).

Nous avons relevé un certain nombre de dictionnaires récents, des glossaires à usage interne et des fichiers-clients des bureaux de traduction.

Certains professionnels se sont attelés à la francisation de l'enseignement de l'informatique. 
Le vocabulaire belge de la vulgarisation informatique accuse du retard par rapport à celui de France. Les traductions des manuels, celles des messages d'écran et des commandes des programmes se sont nettement améliorées au cours de ces dernières années, surtout en micro-informatique (pour les gros systèmes, les sociétés de service en informatique se procurent encore systématiquement la version originale de ces documents). Mais la qualité de certaines revues de vulgarisation laisse encore à désirer, peut-être parce que leurs auteurs et rédacteurs ne sont pas des professionnels de l'écriture comme les journalistes, traducteurs ou philologues. Ces publications accusent notamment les défauts suivants : fautes d'orthographe (sans-abris dans Data News, le marché néerlandophone, Paninformatique); calques sémantiques dans les collocations (dernière actualité dans Data News) et les constructions syntaxiques (Pouvant être étendu sur sept ans, le projet a une durée initiale de trois ans dans Data News). Parmi les erreurs de terminologie, pointons : les imprécisions de vocabulaire; les erreurs, notamment dans les emprunts (des hardwares dans Data News); le manque de cohérence dans la terminologie des différents articles d'un même numéro de revue, voire à l'intérieur du même article (byte voisine joyeusement avec octet et système opératoire avec système d'exploitation).

À l'instigation conjointe du Service de la langue française et de la Taalunie, un gros dictionnaire d'informatique pourrait être élaboré, peut-être en co-édition par une maison d'édition technique wallonne et un éditeur technique flamand. À cette occasion, les bureaux de traduction et les services de traduction des gros constructeurs et des grandes boîtes de logiciels pourraient se voir proposer d'offrir la partie non «maison» de leurs glossaires-clients, après une réunion de sensibilisation destinée à leur montrer que la confidentialité de leurs données ternimologiques est relative...

Une autre mesure importante viserait à réduire l'écart entre la langue française et belge de l'informatique «grand public». Un guide de rédaction d'ouvrages et d'articles (sur le modèle de celui que comporte la norme NBN X 04-002 de l'IBN : Terminologie française. Mises en garde et listes lexicales pour la technique et le commerce, Bruxelles, 1989) ainsi que des numéros spéciaux sur la terminologie pourraient être proposés aux rédacteurs en chef des revues d'informatique belges.

Les représentants de l'industrie rappellent que les milieux belges de l'informatique ou ses diffuseurs n'utiliseront systématiquement le français que quand ils auront un avantage concret à le faire. L'amélioration des services d'un constructeur, par exemple une qualité accrue de la langue de ses manuels, n'est utile que si le client s'en rend compte...

\section{LA TERMINOLOGIE DE LA ROBOTIQUE}

Le vocabulaire de la robotique proprement dite est très restreint : quand on parle des robots, de leur conception et de leurs applications, la terminologie est celle d'autres sciences et techniques, notamment la physique (optique, mécanique), l'électronique, les mathématiques, la logique et l'informatique. Cette interdisciplinarité explique probablement l'absence de besoins en glossaires : la terminologie utilisée se trouve dans les dictionnaires et les glossaires des disciplines connexes.

Le vocabulaire varie selon les niveaux de technicité : recherche, enseignement, application et vulgarisation.

Le vocabulaire de la recherche est très spécialisé. Certains néologismes y apparaissent. La terminologie suit les progrès des disciplines constituant le domaine; actuellement, le vocabulaire informatique et mathématique y occupe une place importante. Si l'anglais est de rigueur au sein des équipes de recherche internationales, ce n'est pas parce que la terminologie serait plus élaborée dans cette langue (les Français conçoivent 
souvent des systèmes), mais parce qu'il facilite la diffusion internationale des nouveaux produits ou des résultats des travaux. Les recherches menées par les équipes nationales le sont dans la langue du pays. Son vocabulaire est relativement bien établi, notamment en français. Les néologismes anglais trouvent assez rapidement des équivalents français.

L'enseignement reprend le vocabulaire de la recherche et des applications. Dans la plupart des institutions, cette terminologie plus large, incluant la terminologie de la mécanique, est en français ; comme la robotique est une discipline neuve, les équivalents français des néologismes anglais sont provisoires. Les monographies et les travaux universitaires sont également en français, excepté un certain nombre de sigles et de noms de commandes. Une partie importante du vocabulaire de la robotique relève de l'informatique, pour laquelle la problématique est tout à fait différente (cf. supra). Presque tous les sigles qui renvoient à des termes anglais relèvent de la terminologie informatique.

Les documents destinés aux ingénieurs contiennent également un vocabulaire multidisciplinaire. Ils sont soit en français (pour la plus grande partie), soit en anglais et quelquefois en franglais.

Les études d'applications charrient surtout le vocabulaire de l'application concrète (assemblage, soudure, peinturage, manutention et palettisation, découpe). Selon la branche ou le client, ce vocabulaire est plus ou moins spécialisé ; il est en français ou dans une autre langue.

Le vocabulaire de la robotique au sens large n'évolue pas très vite; le noyau du vocabulaire s'est stabilisé, tant en français qu'en anglais. L'évolution se fait par à-coups et dans des domaines bien précis : l'invention d'un nouveau système apporte sa moisson de nouveaux termes, notamment de sigles.

Certains néologismes apparaissent dans des documents de vulgarisation.

L'utilisation de l'anglais dans les communications internationales semble inévitable aux responsables des recherches et, selon eux, elle ne compromet pas la francisation du vocabulaire. Voici les arguments qu'ils invoquent à l'appui de leur opinion :

- le règne de l'anglais est dû aux impératifs pratiques de l'intercompréhension au niveau international ;

- ce qui compte, dans la recherche de pointe en général et en robotique en particulier, c'est la rapidité de la circulation de l'information. Or il existe, pour le moment du moins, un décalage structurel entre l'apparition des néologismes anglais et celle de leur équivalent français. Même si ce délai est très court, il n'est pas supportable pour les milieux de la recherche. Or ce délai est irréductible: en supposant que soient traduits tous les actes des colloques importants (ces manifestations se déroulent toujours en anglais, à en croire nos interlocuteurs), ce qui n'est pas le cas, il faudrait attendre la publication de cette traduction française pour voir apparaître les néologismes français ;

- dès le début, les néologismes anglais ont une acception précise. Pour la signification des équivalents français, on observe une période initiale de flottement et, pendant un certain temps, une concurrence entre plusieurs synonymes ;

- l'utilisation de l'anglais n'aurait aucune influence sur la terminologie française, qui existerait en parallèle et serait bien française. Pas de problèmes d'emprunts; on communiquerait soit en français, soit en anglais, mais très peu en franglais. Le français de la robotique ne comporterait que peu d'emprunts à l'anglais, excepté pour le vocabulaire informatique.

La terminologie de la recherche nationale et de l'enseignement est française. C'est ici que manquent les équivalents français pour les néologismes récents. Souvent, il existe déjà des termes utilisés couramment dans l'industrie, mais encore inconnus dans l'ensei- 
gnement. Cette situation n'est pas problématique en soi, parce que tous, étudiants compris, comprennent les termes anglais. La création de néologismes français se fait ici par ceux qui enseignent et poursuivent des recherches. On trouve donc des néologismes français dans les cours et les travaux de recherche. Un certain nombre de néologismes n'ont pas encore trouvé leur équivalent français et un nombre plus important encore d'équivalents français ne sont pas consignés dans les dictionnaires ou bien le consensus ne s'est pas encore fait à leur propos.

Les sigles constituent un problème à part. Ils forment des images visuelles et ont perdu leur motivation. Certains sigles ne peuvent être traduits parce qu'ils représentent des procédés ou des marques protégés. Beaucoup d'interlocuteurs ont suggéré l'adoption d'une politique claire en la matière : si CAD et CAM sont traduits par CAO et FAO, la plupart des sigles sont restés anglais (CIM, VMS, FMS, CIAM, RCC).

L'inventaire révèle que la Belgique a peu de ressources primaires en terminologie de la robotique.

Autant l'inventaire des ressources terminologiques primaires est maigre, autant les possibilités d'activités terminologiques belges semblent importantes. Tout d'abord, les experts en robotique maîtrisent bien l'anglais et le français. Les communications dans ces deux langues sont en outre bien distinctes. De plus, les responsables de l'enseignement désirent la francisation de leur terminologie. Autres ressources secondaires importantes: l'infrastructure documentaire existante et la volonté que manifestent les centres de documentation de consigner et diffuser les néologismes français créés spontanément dans l'enseignement de la robotique industrielle.

\section{LA TERMINOLOGIE DE LA CHIMIE}

Bien que les chimistes communiquent souvent à l'aide de formules, la terminologie chimique est très vaste. Elle sert non seulement aux communications entre chimistes, mais également entre les biologistes, les médecins, les responsables de l'environnement, voire les politiciens et les juristes.

La nomenclature est internationale et très structurée. Elle est normalisée par l'IUPAC (International Union of Pure and Applied Chemistry).

Les chimistes signalent pourtant quelques problèmes en la matière :

- l'existence de nomenclatures différentes (par exemple celle de l'American Chemical Society);

- le fait que la nomenclature ne rend pas exactement la composition des polymères;

- le fait que la nomenclature française en chimie organique s'est voulue longtemps différente de la nomenclature anglo-saxonne et internationale, en particulier dans la position des numéros des substituants attachés au squelette hydrocarboné principal de la molécule 6 . Progressivement, cependant, les chimistes français s'alignent sur la nomenclature internationale.

Les nouveaux produits et les nouvelles molécules se voient attribuer un nom à partir de la nomenclature. La formule chimique peut s'étaler sur plusieurs lignes; on donne dès lors à chaque produit un nom commercial, plus court. À côté de la nomenclature, il existe donc toute une terminologie des molécules et des produits (11 millions de composantes, dont chacune porte un ou plusieurs noms : DDT a une centaine de synonymes).

Le vocabulaire technique regroupe également la terminologie des processus et des réactions, ainsi que celle des différentes théories (atomique, thermodynamique, cinétique, électrotechnique, etc.). Les termes industriels sont interdisciplinaires; ils empruntent à la 
pharmacie, la biologie, etc.) et charrient surtout la terminologie spécifique des applications.

Les revues scientifiques et les actes de colloques sont pour 95 pour cent en anglais. Dans leurs propres revues scientifiques, les chimistes francophones ont presque entièrement abandonné le français. Selon un comptage des publications citées dans le Bulletin signalétique du CNRS et les publications signalées par la base PASCAL du même centre, seulement 4 pour cent des publications sur la chimie sont en français. En Belgique, l'éditorial et la plupart des articles du Bulletin des sociétés chimiques belges sont également rédigés en anglais.

Dans la Belgique francophone, les documents administratifs sont entièrement en français. Les documents commerciaux sont par contre dans la langue du client, donc très souvent en anglais, la chimie couvrant un marché international. Les revues destinées à un public plus large sont en français. Les chimistes signalent que tandis que tous les manuels de l'enseignement secondaire sont en français, les professeurs se réfèrent de plus en plus souvent à des textes anglais dans l'enseignement supérieur.

On peut en conclure que la langue de communication internationale est l'anglais et que toutes les revues scientifiques belges qui veulent avoir une audience internationale contiennent surtout, sinon exclusivement, des articles en anglais. Tous les autres documents, excepté quelques documents utilisés dans l'enseignement supérieur, sont, en Belgique francophone, rédigés en français.

Selon la plupart des chimistes, c'est pour l'obtention d'une audience internationale que l'anglais est nécessaire, et non pour pallier une carence dans la terminologie française, laquelle est aussi élaborée que la terminologie anglaise.

La terminologie chimique française ne semble pas comporter beaucoup d'emprunts directs à l'anglais. Les exemples cités par nos interlocuteurs sont connus: spin (équivalent proposé : toupi); splitting (rotation d'un électron); chemical shift (équivalent proposé : déplacement chimique), etc. Les équivalents français n'ont, selon eux, pas la même précision et presque tous proposent de considérer ces termes anglais comme des termes de chimie universels.

Selon certains chimistes, l'anglais pourrait s'introduire d'une manière indirecte. C'est ainsi que les Français nomment l'étude de nouveaux matériaux nimatérique, dont la racine nim vient de l'expression anglaise New Improved Materials. On remarque également que les noms donnés par l'industrie chimique française aux nouveaux produits ont souvent une connotation anglaise, afin de faciliter leur diffusion sur le marché international. Les chimistes signalent en outre l'apparition récente de néologismes anglais dans la terminologie française des méthodes physiques de l'investigation structurale.

Le vocabulaire français de la chimie est complet et connu. Celui qui veut communiquer en français peut le faire et les chimistes belges francophones le font.

Quant aux traducteurs spécialisés en chimie, ils ne semblent pas connaitre de problèmes majeurs. Grâce à la mise à jour régulière des documents administratifs et scientifiques ainsi qu'à la centralisation de la documentation, ils disposent apparemment de ressources terminologiques suffisantes. D'autant plus que les industriels leur fournissent en général des textes de référence en plusieurs langues. Souvent, les traductions sont d'ailleurs faites dans les entreprises mêmes. Les lacunes importantes relevées par certains traducteurs résultaient de leur propre méconnaissance des documents existants. 


\section{LA TERMINOLOGIE DE L'INDUSTRIE PHARMACEUTIQUE}

Le vocabulaire scientifique traitant des médicaments est interdisciplinaire et international.

Dans l'industrie pharmaceutique, on utilise trois types de dénominations pour les produits: le nom commercial, le nom générique international (INN : International Nonproprietary Name) et le nom chimique.

Le INN ou nom générique est imposé par l'Organisation mondiale de la santé. En français, on connaît aussi un nom générique national, le DCF (Dénomination commune française). Le nom chimique est réglementé par l'IUPAC.

Le vocabulaire est interdisciplinaire et varié parce qu'il suit l'évolution du médicament depuis sa conception jusqu'à sa mise à la disposition du corps médical. Il couvre donc les sous-domaines chimique, galénique, pharmacologique, toxicologique, tératologique, etc., les tests de laboratoire sur l'animal, tant in vitro qu'in vivo, le vocabulaire de l'expérimentation clinique, celui des méthodes d'évaluation statistique, des techniques de production, d'analyse et de contrôle, et enfin le vocabulaire de la commercialisation, de la promotion et de la publicité. Le tout sous-tendu par le vocabulaire médical de base.

La plupart des traductions scientifiques en pharmacie et en pharmacologie sont faites par des médecins et pharmaciens. Il ne semble pas exister de besoins spécifiques à ce niveau, sinon en pharmacologie (tests) ainsi que dans le domaine des éponymes. Les traducteurs traduisent surtout des notices et de la littérature promotionnelle. Cette terminologie ne se trouve pas systématiquement consignée.

En pharmacie, on dispose de documents de référence, multilingues et constamment mis à jour, qui sont utilisés comme dictionnaires ou glossaires par tous les rédacteurs et traducteurs, tels que les Index medicamentorum, pharmacopées, répertoires des médicaments et thésaurus. Le ministère de la Santé publique édite en outre quelques documents terminologiques. La ressource terminologique principale en Belgique est constituée par les fiches techniques qui sont publiées lors de l'introduction de nouveaux médicaments sur le marché belge, ainsi que par les listes de spécialités pharmaceutiques, qui donnent les informations sur la composition, les propriétés et la posologie des médicaments. Ces fiches ou ces listes sont traduites par des pharmaciens et des médecins.

On peut donc conclure que la Belgique semble disposer de ressources terminologiques secondaires suffisantes en pharmacologie et que les vrais spécialistes de la traduction formulent peu de besoins spécifiques.

\section{CONCLUSIONS}

Si on exclut les mémoires de terminologie des étudiants en traduction et les fichiers personnels des traducteurs, l'inventaire de la production belge en terminologie fait apparaître un nombre de vocabulaires relativement réduit, surtout pour certains domaines comme la chimie et l'informatique.

Ce phénomène est sans doute imputable aux faits suivants :

- le nombre assez faible d'éditeurs belges d'ouvrages techniques, notamment de dictionnaires ;

- pour les domaines qui comportent beaucoup de néologismes, une attitude attentiste à l'égard des créations néologiques des scientifiques et techniciens français ;

- l'existence de nombreuses publications utilisables comme terminologies dans certains domaines (listes de produits, pharmacopées, compendiums, etc.).

En comparaison, le nombre de thésauri est plus élevé: les thésauri américains sont souvent traduits en néerlandais et en français pour l'indexation des bibliothèques, 
lesquelles sont souvent bi- ou trilingues, et les documentalistes disposent de temps pour le faire (notamment s'ils travaillent dans les bibliothèques d'organismes publics et de centres de recherche). Il faut noter que l'informatisation des bibliothèques pourrait entraîner à moyen terme la disparition de ce type de ressources et son remplacement par les index automatiques d'ouvrages (celui des projets de recherches universitaires belges des Services de la programmation scientifique du premier ministre). Comme outils d'aide aux recherches ponctuelles de terminologie, ces index automatiques présentent le désavantage de n'être ni structurés ni lemmatisés.

L'identification des vocables belges est malaisée :

- les banques de données documentaires (entre autres PASCAL) et les bibliographies sont axées sur les publications françaises ;

- le dépôt légal belge n'est pas à jour et les éditeurs oublient souvent d'y déposer un exemplaire de leurs publications ;

- les auteurs et traducteurs belges travaillant pour des éditeurs étrangers, notamment hollandais ou français, sont difficiles à identifier (les informations sur les auteurs sont confidentielles).

La qualité des documents répertoriés est très inégale. Les mémoires d'étudiants sont de qualité variable. Certains glossaires destinés au grand public ne comportent que les termes de base de la discipline ${ }^{7}$. Parmi eux, signalons des vocabulaires encyclopédiques (comme celui de Télindus, sur les télécommunications), dont les termes anglais sont présentés sans traduction, avec une définition en anglais et en néerlandais. D'autres sont très techniques 8 .

On ne s'étonnera pas de la place qu'occupe le néerlandais dans ces ouvrages. L'allemand y apparaît souvent.

Il existe des publications commerciales dans chacun des domaines examinés. En chimie et en pharmacologie, les catalogues de produits sont des terminologies. Les documents promotionnels (publicités, revues de prestige) et contractuels sont souvent traduits; le rôle des anglicismes que comportent nombre de documents publicitaires ou de prestige est souvent cryptique. Dans des activités réglementées comme la pharmacologie et la construction, où la sécurité du public est en jeu, les ministères diffusent ou imposent des documents prescriptifs, généralement en français et en néerlandais; certains d'entre eux constituent des terminologies ou prescrivent l'usage de vocables: le commission d'agréation des médicaments impose une terminologie pour les notices destinées au public ; la définition des nouveaux matériaux de construction est agréée ou normalisée en français et en néerlandais. En informatique, par contre, tous les documents sont d'origine privée. Les monopoles y sont une source d'harmonisation du vocabulaire (télématique).

Les disciplines anciennes (chimie) et «locales» (construction) sont plus françaises que certaines disciplines récentes (informatique), qui se sont développées d'emblée à l'échelon international. La langue peut aussi varier selon les sous-domaines: la nomenclature est en «international» (confixes), les noms de produits sont en anglais et les analyses sont en français. Les domaines interdisciplinaires (robotique, pharmacologie et, à un degré moindre peut-être, construction) posent plutôt des problèmes de polysémie que de statut des langues.

Au sein d'un même domaine, la langue peut varier d'après le produit industriel sur lequel portent les documents techniques: en informatique, les lettres d'annonce, les manuels d'entretien ou d'utilisation, les messages d'écran, même, sont entièrement en anglais pour les gros systèmes, alors que pour les micro-ordinateurs, ces textes sont traduits dans toutes les langues.

Les publications scientifiques des cinq domaines sont très similaires: actes de colloques, comptes rendus de conférences, revues, manuels et monographies. Dans tous 
ces textes, l'usage de l'anglais est de règle à l'échelon international, où les publicationsphares sont américaines et où la communication doit être rapide. En Belgique, les chercheurs communiquent entièrement en français dans les disciplines anciennes comme la chimie, l'architecture et la construction, en franglais (informatique) ou en français et en anglais alternativement (robotique, dans les activités récentes). L'informatique et la pharmacie charrient des documents de vulgarisation nombreux : manuels d'utilisation de progiciels pour la première, notices de médicaments pour la seconde. La qualité de la langue de ces documents varie (très française pour les notices, relâchée et anglicisée pour les revues de vulgarisation de l'informatique). Dans les disciplines anglicisées, les notes de cours comportent souvent une terminologie bilingue: le terme français est accompagné de son équivalent anglais, placé entre parenthèses.

L'évolution du vocabulaire peut être continue ou se faire par à-coups, lorsque les découvertes se multiplient dans un secteur précis. Le rythme d'évolution du vocabulaire varie d'un domaine et parfois d'un sous-domaine à l'autre: le vocabulaire de la toiture en chaume est relativement stable, celui de l'intelligence artificielle est en mutation rapide. L'informatique montre que le vocabulaire spécialisé se francise - et se «déterminologise» pour une part, c'est-à-dire que des termes disparaissent - lorsqu'il se vulgarise. Certaines étapes de l'évolution des firmes sont l'occasion de changements importants pour leur vocabulaire. Ce fut le cas de l'harmonisation des produits de la gamme SAA d'IBM (architecture de réseau unifiée), qui a entraîné la création et l'imposition par la société d'une terminologie pour toute la gamme des produits compatibilisés par cette nouvelle structure.

La production de dictionnaires est plus marquée dans les domaines où l'apport des autres pays est inexistant (droit, certains secteurs de la construction). On trouve particulièrement peu de vocabulaires dans les domaines où l'autogestion des vocables par les spécialistes est efficace (chimie). Des terminologies de vulgarisation sont publiées dans des domaines où le vocabulaire est une entrave directe à la communication avec le grand public (lexique des télécommunications, lexique hospitalier). Les termes des domaines interdisciplinaires sont déjà consignés dans des dictionnaires faits pour les spécialités qu'englobent ces domaines, spécialités dont l'extension est plus large ou l'importance économique plus grande que le domaine en question. Aussi les vocabulaires sont-ils peu nombreux dans ce type de discipline (robotique, pharmacie). Les thésauri complètent les dictionnaires dans les domaines où la production documentaire est importante (construction).

Pour chaque domaine, nous avons cité un certain nombre de personnes et d'entités qui peuvent être considérées comme des ressources au sens large. Elles se recrutent parmi les experts intéressés par les questions de vocabulaire, soit par goût (de la collection, entre autres!), soit pour des motifs professionnels (rédaction de cours, notamment). L'étude de la situation dans cinq domaines et nos coups de sonde dans les associations, fédérations et autres unions confirment que ce sont surtout les centres de recherche, associations professionnelles, universités, services d'agréation (au sens large) des ministères, groupes de pression (associations des producteurs de tabac, ligue contre le cancer, etc.) qui peuvent s'avérer utiles comme partenaires.

Dans l'optique gestionnelle prônée par les spécialistes et les diffuseurs de terminologie, la présente enquête souligne la sujétion incontestée de la terminologie belge au vocabulaire de France, y compris, à un degré un peu moindre, dans les domaines où le savoir-faire belge est développé ou ancien. Les différences qu'accuse notre français technico-scientifique avec celui de la France sont un simple décalage temporel, dans l'assimilation des emprunts à l'anglais notamment. La première réaction de recul des francophones belges, devant un néologisme cré selon les lois de la langue française, 
manifeste la résistance au changement linguistique typique des pays situés à la périphérie d'une langue: peu après, les mêmes personnes adoptent l'équivalent français, oubliant complètement leur mouvement initial de rejet.

Pour les savoirs et savoir-faire assez anciens, les besoins de consignation se réduisent généralement à une mise à jour des meilleurs ou des seuls dictionnaires existants. Souvent, ces mises à jours existent: les traducteurs ajoutent au crayon les termes qui manquent dans les dictionnaires qu'ils consultent souvent, mais ce travail n'est pas diffusé... Pour certains domaines, des besoins explicites de dictionnaires ont été formulés en Belgique, souvent pour la combinaison linguistique français/néerlandais (vocabulaire de l'informatique).

Dans les secteurs très «internationaux» (télécommunications), c'est uniquement l'explication des concepts qui est souhaitée, notamment pour la vulgarisation. Aussi peu orthodoxe qu'elle puisse paraître, une publication rapide comportant des définitions en français de concepts anglais peut rendre de grands services. Les professeurs, notamment d'université, ont des ressources à offrir en la matière, comme le montre le travail minutieux de comparaison des définitions consignées dans la littérature pour les concepts de construction enseignés à la faculté d'ingénieurs en architecture de l'Université de Liège.

Le décalage entre l'apparition des termes anglais et le consensus sur un équivalent français, d'une part, l'intervalle entre ce nouvel usage et son adoption en Belgique, d'autre part, sont structurels. Ces deux phénomènes entraînent en Belgique un flottement de l'usage et un ancrage plus grand des anglicismes. Les spécialistes le déplorent ou continuent à utiliser des termes anglais à cause de lui. Les politiques de la langue peuvent se fixer pour objectif la réduction de ce double intervalle (non sa disparition, sans doute). Des demandes claires d'intervention en ce sens nous sont parvenues. Les traducteurs sont notamment pris entre deux feux : ils doivent à la fois éviter d'être accusés de francisation à outrance («à la française», entendez : «artificielle») et d'incompétence en traduction, un risque auquel ils s'exposent s'ils émaillent leurs textes d'anglicismes (pour rester «belges»); tout nouveau client est un coup de dé à cet égard... Des professeurs désireux d'enseigner leur spécialité en des termes français voudraient, quant à eux, éviter de créer des néologismes «dialectaux».

La présente recherche a fait apparaître l'importance de la documentation technicoscientifique. Certains documents spécialisés sont capitaux pour la néologie parce qu'ils diffusent les nouvelles notions scientifiques. Certains de ces documents sont en outre des dictionnaires en bonne et due forme, parfois inconnus des traducteurs...

Il existe donc en Belgique des ressources spécifiques en matière de néologie française, et il est possible de systématiser les activités néologiques belges. Les rédacteurs techniques, les chercheurs, l'industrie et les enseignants sont conscients de la dispersion de leurs efforts ainsi que du parallélisme de leurs collègues québécois, français et suisses. 
Notes

1. B. Thouin, conférence non publiée.

2. IBM System Journal décrit par exemple les recherches d'IBM dans les domaines de pointe.

3. (Language for Special Purposes): limitation des performances à la lecture, à la compréhension des textes de spécialité et aux conversations à leur propos.

4. «Le vocabulaire médical: jargon ou argot?», La Linguistique, Société internationale de linguistique fonctionnelle, vol. 22, fasc. 1, Paris, 1986.

5. La règle consistant à rendre toutes les comnotations esthétiques, affectives et sociales du message original et son contenu avant sa forme.

6. Considérant ce numéro comme un adjectif, les Anglo-Saxons le placent avant le nom, mais les Français après, ce qui posait des problèmes pour la consultation des dictionnaires et autres listes.

7. Dictionnaire d' informatique de Marabout, etc.

8. Glossaire de la robotique de la maison de M. Scheen. 